\title{
A PRIORI ESTIMATES, GEOMETRIC EFFECTS AND ASYMPTOTIC BEHAVIOR
}

\author{
BY FRITZ JOHN
}

Many physical phenomena can be described by a partial differential equation $P u=0$. Here $P$ denotes some differential operator or system of such operators, and $u$, the unknown function, is either a scalar or a vector. The differential equation connects the derivatives of $u$ at each point of its domain $D$. The mathematician is interested in the global consequences of this local constraint, especially those leading to a better understanding of the physical processes described. There are many different angles from which to look at this subject. In this talk I intend to discuss some particular ones that have to do with the use of a priori inequalities, either implied by the differential equation or postulated for $u$. Some general remarks will lead up to their use.

Naturally, the first impulse of a mathematician on being confronted with an equation $P u=0$ is to solve it. Usually, ${ }^{1}$ the equation is not sufficient to determine $u$, and we have a whole infinite family $S$ of solutions $u$. To characterize the individual members of $S$, we need additional pieces of information, data $f$ taken from a family $\phi$. Ideally, we generate the general element $u$ of $S$ by a continuous 1-1 mapping $T: \phi \rightarrow S$. The "problem" of constructing $u$ from $f$ is then "correctly-set" or "well-posed" for the equation $P u=0$ in the sense of Hadamard [3], [4]. The best known example is the Laplace equation $\Delta u=0$ in a bounded domain $D$ with continuous boundary values $f$ prescribed on the boundary $B$ of $D$. Such a mapping of data $f$ onto solutions $u$ not only has an esthetic appeal, but in many cases a physical interpretation as well, in which $f$ and $u$ somewhat play the roles of cause and effect, indicating perhaps some pre-established harmony between mathematics and the physical world.

Progress in the solution of well-posed problems has been spectacular. Still, this should not blind us to the fact that in applications the role of the well-posed problem is very limited (see [5]). In most cases, the assumption that we have adequate knowledge of the data $f$ to generate $u$ is fiction. Moreover, generating the solution $u$ by solving some particular well-posed problem may contribute very little to a real understanding of the constraint $P u=0$, beyond the insight that nice $f$ produce nice $u$. One might even say that the better behaved the solution of a problem, the more likely it is to be devoid of qualitative features the mind can get hold of. To produce some

Josiah Willard Gibbs Lecture, given under the auspices of the American Mathematical Society, January 23, 1975. The research was supported in part by National Science Foundation grant NSF-GP-36992X2; received by the editors February 28, 1975.

AMS (MOS) subject classifications (1970). Primary 35B45, 35B40.

${ }^{1}$ But not always, as found by H. Lewy; see [1], [2]. 
contrast in this gray featureless landscape of solutions it is useful to consider extreme situations, that amplify some effects. For an elementary illustration, we might think of the problem of solving $n$ linear algebraic equations $\sum_{k=1}^{n} a_{i k} x_{k}=y_{i}, i=1, \cdots, n$, in $n$ unknowns $x_{k}$, which becomes of theoretical interest only when the determinant is zero or very small. In our case, extreme situations correspond to some degeneracy either in the solution $u$, or in the operator $P$, or in the domain $D$. Such degeneracies bring out striking new types of behavior, often concentrated along surfaces and leading to simple geometric interpretations. This behavior is concealed in the general solution $u$, which represents a compromise between various extremes. A good deal of mathematical physics consists of an analysis of the asymptotic behavior produced by extreme conditions of various kinds, such as shock waves in gases [6], [7], electromagnetic waves of high frequency (leading to "geometrical optics" [8]), very steep waves on the surface of water ("bores" [9]), flows of liquids of very low viscosity [10], or deformations of very thin elastic rods [11].

In deriving asymptotic behavior, early pioneers relied as much on physical intuition as on mathematical arguments, neglecting some quantity here but not there. Now, in principle, the differential equations (if they are to be trusted) already contain information on all possible situations, and any asymptotic behavior should follow from those equations without any appeal to intuition. Such a rigorous deduction is not just an exercise in logic. Physical intuition may fail, and even where it does not, it is only mathematical analysis that can tell us the exact circumstances in which the asymptotic behavior occurs.

During the last decades, applied mathematicians have been highly successful in reviewing and extending many classical results (some going back more than a century), and in initiating new lines of investigation. They have been helped by advances made in the theory of partial differential equations, particularly in estimating solutions. Asymptotic behavior ordinarily depends on the fact that certain terms in the equation $P u=0$ become negligible, while others can be combined with certain of the boundary conditions to yield a new asymptotically valid system of equations $P^{\prime} u=0$. Now, the mere fact that the coefficient of some derivative is small does not imply that the whole term is small, unless we somehow restrain the growth of derivatives. Here is where we need a priori estimates that permit us to decide on the relative orders of magnitude of the various derivatives of $u$ occurring in $\mathrm{Pu}$.

Estimating is the central activity in the theory of partial differential equations; it serves to justify both abstract existence proofs and numerical computations. Estimates, as often presented in a string of lemmas, may look singularly unattractive, lacking the elegance of giving the best constants, and merely concerned with orders of magnitude. They do, however, express deep truths and lead to results not easily obtainable by algebraic manipulations of the differential operators. The most complete estimates exist for differential operators $P$ of the type called elliptic, the ones usually encountered in the description of equilibrium states [12], [13], [14]. For such $P$, one 
can estimate the derivatives of $u$ in terms of $P u$ and $u$ or of $P u$ and boundary data $f$, using a variety of norms. For example, for a solution $u$ of the $n$-dimensional Laplace equation $\Delta u=0$ in a domain $D$, we have for any $k$ th order derivative the Cauchy estimate

$$
\left|u^{(k)}(x)\right| \leqq\left(\frac{2 \Gamma((n+2) / 2)}{\pi^{1 / 2} \Gamma((n+1) / 2)} \frac{1}{d(x)}\right)^{k} U
$$

where $d(x)$ denotes the distance of the point $x$ from the boundary $B$ of $D$, and $U$ is the supremum of $|u|$ in $D$. For more general linear (and semilinear) elliptic equations $P u=0$, we have similar looking estimates of the form

$$
u^{(k)}(x)=O\left(U /(L(x))^{k}\right)
$$

where $U$ and $u^{(k)}$ have the same meaning as before, and a suitable "wave length" $L(x)$ can be computed a priori in terms of the distance $d(x)$ from the boundary $B$ and of "representative lengths" $\lambda, \mu$ associated with the operator $P$ by the formula

$$
L(x)=\lambda d(x) /(\mu+d(x)) .^{2}
$$

Formula (3) reflects the decay of outside influences on the solution $u$ with increasing distance from the boundary. For $d \gg \mu$, the wave length $L$ is of the order of $\lambda$; for $d \ll \mu$ it is essentially of order $d$, leading to a break down of the estimate (2), as $x$ approaches the boundary. ${ }^{3}$

As an example, we consider the scalar two-dimensional equation

$$
P u=h^{2} \Delta^{2} u-T \Delta u=0
$$

which expresses the equilibrium condition for the normal displacement $u$ of a plate of thickness $h$ under uniform tension $T$, when no forces act on the faces of the plate. The variable $x=\left(x_{1}, x_{2}\right)$ varies over the domain $D$ with boundary $B$. Here, in accordance with dimensional analysis, the only length associated with the operator $P$ is $\lambda=h / \sqrt{ }|T|$. Accordingly, we have an estimate of the form (2) with

$$
L=\lambda d(x) /(\lambda+d(x)) \sim \operatorname{Min}(\lambda, d(x)) .
$$

For $h \rightarrow 0$, one would expect equation (4) to yield asymptotically the membrane equation $\Delta u=0$. Usually, this transition from plate to membrane is discussed in the framework of the theory of singular perturbation of operators, representing $u$ as solution of a well-posed problem $u=T_{h} f$ in terms of data $f$, and discussing the limit $h \rightarrow 0$ in appropriate function

\footnotetext{
${ }^{2}$ The estimate (2) is not supposed to hold uniformly in $k$ for all $k$, but only for $k$ bounded by some fixed number $K$, the coefficients of $P$ having sufficiently many continuous derivatives. In the semilinear case, moreover, $U$ is assumed to be sufficiently small. The lengths $\lambda$ and $\mu$ depend on bounds for the coefficients of $P$ and their derivatives, and on the modulus of ellipticity in $D$. Estimates of type (2) follow, for example, from the explicit integral representation for $u^{(k)}$ in terms of $u$ given in [15], [16].

${ }^{3}$ For the Laplace operator, which contains no representative length, $L$ reduces to $d$.
} 
spaces. ${ }^{4}$ It is simpler just to apply estimates of type (1) provided we are willing to completely ignore the boundary conditions as well as the boundary behavior, and instead postulate knowledge of only an upper bound for the supremum $U$ of $|u|$ in the whole domain $D$. The general approach here is motivated by the expectation that interior asymptotic behavior should be quite independent of the specific boundary conditions imposed. While, in principle, the required upper bound for $U$ could be derived from a sufficiently detailed knowledge of the boundary conditions, this may be irrelevant for many purposes, where $U$ just enters as a convenient and easily controlled norm for $u$, against which the deviation from asymptotic behavior for $u$ can be measured. It turns out now that the estimate (2) with the choice $L=\operatorname{Min}(\lambda, d(x))$ is insufficient to yield the transition from plate to membrane for $h \rightarrow 0$. As a matter of fact, for $T<0$ the growth of the fourth derivatives in equation (4) cannot be controlled, and thin plates under compression cannot be expected to behave like membranes. It is only for $T>0$ that the approximate validity of the asymptotic equation $P^{\prime} u=\Delta u=0$ can be justified on the basis of refined derivative estimates, ${ }^{5}$ which show that here (2) still holds with the stronger choice

$$
L=\operatorname{Min}\left(\lambda e^{\mathrm{d} / 2 \lambda}, d e^{\mathrm{d} / 2 \lambda}\right), \quad \lambda=h / \sqrt{ } T .
$$

For purposes of illustration, take the case where $D$ is a circular disk of radius $r \gg \lambda$, and $D^{\prime}$ the concentric disk of radius $r / 2$. For $x \in D^{\prime}$, we have $d(x) \geqq r / 2 \gg \lambda$, and thus by (6) $L(x) \geqq \frac{1}{2} r e^{r / 4 \lambda}$. We conclude from (2) and (4) that in $D^{\prime}$

$$
\Delta u=\lambda^{2} \Delta^{2} u=O\left(\lambda^{2} L^{-4} U\right)=O\left(U \lambda^{-2} e^{-r / \lambda}\right) .
$$

Taking for $v$ the solution of the membrane equation $P^{\prime} v=\Delta v=0$ with the same values as $u$ on the boundary of $D^{\prime}$, we find from the maximum principle that

$$
u-v=O\left(r^{2} \Delta(u-v)\right)=O\left(U r^{2} \lambda^{-2} e^{-r / \lambda}\right)=O\left(U e^{-r / 2 \lambda}\right) .
$$

We see that in $D^{\prime}$ the plate solution $u$ differs from a membrane solution $v$ by an amount that is small compared to the supremum $U$ of $|u|$ in $D$, provided the quantity $r / \lambda=T^{1 / 2} r / h$ is large. This constitutes a more precise description of the interior limiting behavior of $u$ for $h \rightarrow 0$.

In the preceding example, it is the operator $P$ that degenerates. For applications in which the domain $D$ degenerates, say becomes very thin, formula (3) for the wave length is insufficient for deriving asymptotic behavior, since, in a thin region, we are unfortunately everywhere close to the boundary $B$. Here, more refined global estimates can be applied, when on a portion $B^{\prime}$ of the boundary, suitable "complementing" homogeneous boundary conditions $\Lambda u=0$ are satisfied [26]. We can then ignore the location of $x$ relative to $B^{\prime}$, and replace $d(x)$ in formula (3) for $L(x)$ by the distance $d^{\prime \prime}(x)$ from the remaining boundary portion $B^{\prime \prime}=B-B^{\prime}$ (possibly

\footnotetext{
${ }^{4}$ See [17] [22]. A very complete analysis, including boundary layer expansions for the general nonlinear v.Karman-Föppl plate equations, has been given by P. Fife [23].

${ }^{5}$ Easily verified using the decomposition $u=v+w$, where $\Delta v=0, h^{2} \Delta w-T w=0$. For an analogous treatment of more general plate equations, see [24], [25].
} 
modifying the constants $\lambda, \mu$ to take into account any representative lengths associated with $B^{\prime}$ ). Moreover, at points $x$ of $D$ for which the distance $d^{\prime}$ from $B^{\prime}$ is small compared to $L(x)$, the condition $\Lambda u=0$ can be expected to hold approximately and to lead, when combined with $P u=0$, to a new asymptotically valid system $P^{\prime} u=0 .^{6}$

As an elementary example, consider the Cauchy-Riemann equations $u_{x}=v_{y}, u_{y}=-v_{x}$ in a rectangle $D:|x|<a,|y|<h$, with the boundary condition $v=0$ imposed on the portion $B^{\prime}:|y|=h,|x|<a$ of the boundary. We can interpret $(u,-v)$ as the velocity field of a flow through a channel of width $2 h$ and length $2 a$, with liquid entering or leaving at the ends $B^{\prime \prime}:|x|=a,|y|<h$. One can show that for $h \ll a$ the flow is approximately uniform as long as we keep away from the ends. For that purpose, introduce $U=\sup _{D}|u|$. By reflection, $u, v$ can be continued immediately into the whole strip $|x|<a$ without changing the differential equation or the bound $U$. Since here $d^{\prime \prime}=a-|x|$ is the distance of $(x, y)$ from the boundary of the strip, we successively find from the Cauchy estimates (1) and the boundary condition that $v_{y}=u_{x}=O\left(U / d^{\prime \prime}\right), v=O\left(U h / d^{\prime \prime}\right), u_{x}=v_{y}=O\left(U h / d^{\prime \prime}\right), u_{y}=-v_{x}=$ $O\left(U h / d^{\prime \prime 2}\right)$. Hence, we find for example, that in the subdomain $|x|<a / 2$, $|y|<h$,

$$
v=O(U h / a), \quad u-u(0,0)=O(U h / a),
$$

so that for $h \ll a$ the flow is approximately uniform in the central section of the channel. ${ }^{7}$ Details of the velocity distribution at the ends are filtered out in the interior.

A very similar situation (though much harder to discuss) is presented by the classical principle of Saint-Venant (1853) for the equilibrium deformation of a long elastic cylindrical column subject to forces acting at the ends. The condition that no forces act on the lateral portion $B^{\prime}$ of the boundary implies that at each point of $B^{\prime}$ certain combinations of components of the stress tensor vanish. St. Venant's principle requires these same combinations to vanish effectively throughout the cylinder except near the ends, leading to the observation that all details of the force distribution at the ends, except for the resultant and resultant moment, are filtered out in the interior. An actual proof of the principle ${ }^{8}$ was given only some 10 years ago through the work of R. A. Toupin [28] and (in two dimensions) J. K. Knowles [29] and J. Roseman [30]. Here again, the formulation of the principle involves an a priori bound either on the total strain energy or the maximum stress. Similarly, the interior asymptotic behavior of a thin elastic shell in equilibrium can be derived on the basis of a postulated a priori bound for the maximum stress [31].

Everything said so far applies to elliptic equations (or also to hypoelliptic ones [2], [32]). In the case of hyperbolic equations, or others, describing

\footnotetext{
${ }^{6}$ It is plausible that $L$ is also a wave length for $\Lambda u$, so that $\operatorname{grad} \Lambda u=O\left(L^{-1} \sup _{\mathrm{D}}|\Lambda u|\right)$. Since $\Lambda u$ vanishes on $B^{\prime}$, one can then expect that $\Lambda u=O\left(d^{\prime} L^{-1} \sup _{D}|\Lambda u|\right) \ll \sup _{D}|\Lambda u|$ in points for which $d^{\prime}(x) \ll L(x)$.

${ }^{7}$ Of course, much stronger estimates hold.

${ }^{8}$ And incidentally a correct formulation.
} 
general time dependent processes, there is no possibility of estimating higher derivatives in terms of lower ones. Irregularities do not necessarily get smoothed out, but, on the contrary, can arise spontaneously. Thus, for example, there is no filtering effect as in Saint-Venant's principle that applies to elastic waves travelling along a long cylinder. Hence, in justifying asymptotic behavior, more a priori bounds may have to be postulated. A recent example is the derivation by $\mathrm{N}$. Berger [33] of the classical Boussinesq and Korteweg-DeVries equations for shallow water flow.

In conclusion, I should like to discuss asymptotic behavior of a rather different type associated with a degeneracy in a certain overdetermined system of partial differential equations, and with the notion of "quasiisometric mappings". A deformation or mapping of a domain $D$ in $R^{n}$ can be represented by a vector $u: D \rightarrow R^{n}$. We denote by $u^{\prime}(x)$ the matrix of its first derivatives (the "Fréchet derivative" of $u$ ). The relative changes in line element (or relative changes in distances between "neighboring" points) under the mapping can be described by the strain matrix $e$ defined by the quadratic formula

$$
e=\frac{1}{2}\left(u^{\prime T} u^{\prime}-I\right)
$$

where $I$ is the unit matrix and $T$ denotes transposition. For the simplest elastic materials, there are constituent laws connecting $e$ with the stress tensor, and leading to equations of equilibrium or of motion [34], [35] in terms of $u$ and $e$. The simpler classical linear theory of elasticity derived from these is based on the two assumptions that $e$ is "small" and that $u^{\prime}$ deviates "little" from a constant value throughout $D$. Physically, the first assumption amounts to a restriction to small stresses, while the second, more doubtful one, has the character of a global geometric constraint. To what extent does the first assumption imply the second one? For $e=0$, the mapping $u$ is rigid, and $u^{\prime}$ is equal to a constant orthogonal matrix. However, experience shows that small strains $e$ are quite compatible with large variations in $u^{\prime}$ (large relative "rotations" of different parts of $D$ ). This occurs particularly when we deal with thin rods or shells (that is with degenerating domains $D$ ), and accounts, in fact, for some physical phenomena (like "buckling") that are specifically nonlinear. Now, from (7), $u^{\prime \prime}$ can be expressed in terms of $u^{\prime}, e^{\prime}, e$ ("Christoffel relations") in a way that shows that small strains $e$ and small strain rates $e^{\prime}$ imply small $u^{\prime \prime}$, and hence (to a degree depending on the shape of $D$ ), nearly constant $u^{\prime}$. In the case where the deformation $u$ corresponds to an equilibrium state in the absence of volume forces, the strain $e$ is a solution of an elliptic system, and hence, by (2), small $e$ yield only correspondingly small $e^{\prime}$, and $u^{\prime}$ will indeed be nearly constant, except possibly near the boundary $B$ of $D$ where (2) breaks down. However, in the absence of an elliptic system for $e$ (as in elastic body motion), a bound on $e$ supplies no information on $e^{\prime}$ or $u^{\prime \prime} .{ }^{9} \mathrm{We}$

\footnotetext{
${ }^{9}$ For dimensional reasons, a postulated a priori bound for $e^{\prime}$ would be of a different physical character than a postulated bound for the dimensionless $e$, since a bound for $e^{\prime}$ would be tied to the introduction of a representative length.
} 
now have the remarkable fact that bounds on $e$ alone already imply bounds on the mean oscillation of $u^{\prime}$.

Mappings $u$ with $|e|<\varepsilon$ in the domain $D$ are called $\varepsilon$-quasi-isometric [36], [37], [38]. A typical simple example is furnished by the plane mapping described in polar coordinates by

$$
(r, \theta) \rightarrow(r, \theta+\varepsilon \log r),
$$

for which

$$
u^{\prime}=\left(\begin{array}{cc}
\cos (\varepsilon \log r) & -\sin (\varepsilon \log r) \\
\sin (\varepsilon \log r) & \cos (\varepsilon \log r)
\end{array}\right)+O(\varepsilon), \quad e=O(\varepsilon) .
$$

The mapping preserves circles about the origin, rotating them by an amount depending on their radius (Figure 1 indicates this mapping for $\varepsilon=1$ with the unit disk as domain $D$, showing the image of the coordinate grid in the $x_{1} x_{2}$ plane). This example illustrates several conclusions that can be made for the general quasi-isometric mapping with small $\varepsilon$ in any dimension. First of all, we see from (9) that $u^{\prime}$ is not everywhere close to a constant matrix but comes close to every proper orthogonal matrix. Moreover, this high variability of $u^{\prime}$ is not confined to a neighborhood of the boundary of $D$, but is most pronounced right at the center. ${ }^{10}$ (It is easy to make up other examples of $\varepsilon$ quasi-isometric with large oscillations of $u^{\prime}$ scattered throughout the domain $D$, though only in a set of small measure.) For small $\varepsilon$, the image under $u$ of any cube $C$ of side $h$ contained in $D$ is approximately a congruent cube, though, in detail, the mapping of $C$ can be far from rigid. (This is shown in Figure 2, an enlarged detail of Figure 1, showing the image of a coordinate square with vertex at the origin). More precisely, if $\gamma_{c}$ is the average of $u^{\prime}$ in $C$ and $\beta_{c}$ a suitable constant vector, the estimate $u-\gamma_{C} x-\beta_{C}=O(\varepsilon h)$ is valid throughout $C$. However, the average $\gamma_{C}$ (represented in Figure 1 by the angle by which the original coordinate squares $C$ have turned) varies according to the size and location of $C$, and the shape of the domain $D$. For a domain $D$ that is convex and whose boundary lies between two concentric spheres of radii $a$ and $b(a<b)$, one finds that

$$
\gamma_{C}-\gamma=O(\varepsilon(b / 2) \log (1+a / h)),
$$

where $\gamma$ is the average of $u^{\prime}$ in $D$. Moreover, in the points of $D$,

$$
u-\gamma x-\beta=O\left(\varepsilon b^{2} / a\right)
$$

for a suitable constant $\beta$. Formula (11) shows that the kinematic rigidity of the convex domain $D$ under deformations with small strain, is higher for balls $(a=b)$ than for elongated solids $(a \ll b)$.

The preceding discussion involved the average $\gamma_{C}$ of the derivative of an $\varepsilon$-isometric mapping. We can show more precisely that the mean oscillation

\footnotetext{
${ }^{10}$ This shows incidentally that the "twisting" deformation (8) cannot represent an equilibrium position for an elastic body unless it is one maintained by a suitable external "volume force".
} 


\section{$(r, \theta) \rightarrow(r, \theta+\log r)$}

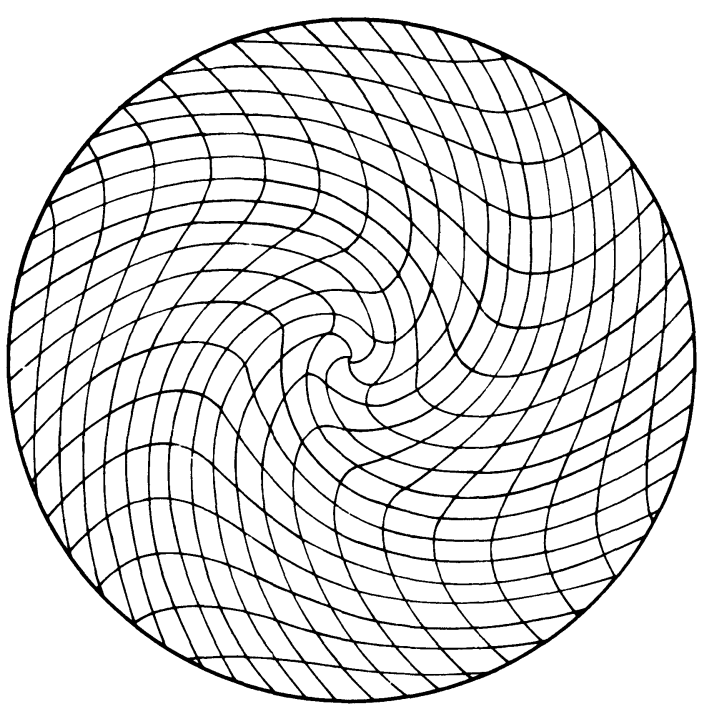

FIGURE 1

of $u^{\prime}$ (see [39]) is of order $\varepsilon$, that is that

$$
h^{-n} \int_{C}\left|u^{\prime}-\gamma_{C}\right| d x=O(\varepsilon)
$$

for each cube $C$ in $D$. This can be shown to imply that, more generally, for any $p$ with $1 \leqq p<\infty$,

$$
\left(h^{-n} \int_{C}\left|u^{\prime}-\gamma_{c}\right|^{p} d x\right)^{1 / p}=O(p \varepsilon) .
$$

Thus, in every cube $C$, the function $u^{\prime}$ differs in the $L_{p}$-sense from a constant by an amount of order $\varepsilon$ as long as $p$ is finite.

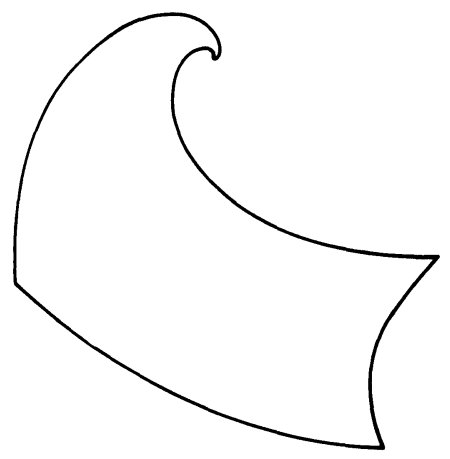

FIGURE 2 
The curious family of functions of bounded mean oscillations, introduced in [39], has been applied and studied extensively (see [40]-[46], and the references given there). More recently, it has been linked to another class of mappings $u$ characterized by an a priori inequality for $u^{\prime}: H$. M. Reimann [47] proves that the logarithm of the Jacobian determinant of a quasiconformal mapping is of bounded mean oscillation.

There are still other types of questions that take on a new meaning when we impose an a priori bound on the unknown $u$ (assuming that one has $a$ priori reasons for the belief that there might exist a solution $u$ with such a bound). This situation arises in connection with some improperly posed problems (for example, Cauchy problems for analytic nonhyperbolic equations, or problems involving analytic continuation) where $u$ is uniquely determined in terms of data $f$, but where the operator $T$ generating $u$ from $f$ is not continuous [48], [5]. Here, imposition of a bound $|u| \leqq U$ may regularize the problem. The restriction of $T$ to the subfamily described by the inequality can be continuous, and this may even make it possible to determine $u$ from $f$ by a suitable stable numerical scheme.

\section{REFERENCES}

1. H. Lewy, An example of a smooth linear partial differential equation without solution, Ann. of Math. (2) 66 (1957), 155-158. MR 19, 551.

2. Lars Hörmander, Linear partial differential operators, Die Grundlehren der math. Wissenschaften, Band 116, Academic Press, New York; Springer-Verlag, Berlin, 1963. MR 28 \#4221.

3. Jacques Hadamard, Lectures on Cauchy's problem in linear partial differential equations, Yale Univ. Press, New Haven, Conn., 1923.

4. R. Courant and D. Hilbert, Methods of mathematical physics. Vol. II: Partial differential equations, Interscience, New York, 1962. MR 25 \#4216.

5. L. E. Payne, Improperly posed problems in partial differential equations (to appear).

6. R. Courant and K. O. Friedrichs, Supersonic flow and shock waves, Interscience, New York, 1948. MR 10, 637.

7. G. B. Whitham, Linear and nonlinear waves, Wiley, New York, 1974.

8. M. Kline and I. W. Kay, Electromagnetic theory and geometrical optics, Pure and Appl. Math., vol. 12, Interscience, New York, 1965. MR 31 \#4330.

9. J. J. Stoker, Water waves: The mathematical theory with applications, Pure and Appl. Math., vol. 4, Interscience, New York, 1957. MR 21 \#2438.

10. R. E. Meyer, Introduction to mathematical fluid dynamics, Wiley, New York, 1971.

11. A. E. H. Love, A treatise on the mathematical theory of elasticity, reprint, Dover, New York, 1944. MR 6, 79.

12. L. Nirenberg, Estimates and existence of solutions of elliptic equations, Comm. Pure Appl. Math. 9 (1956), 509-529. MR 19, 962.

13. S. Agmon, Lectures on elliptic boundary value problems, Van Nostrand Math. Studies, no. 2, Van Nostrand, Princeton, N.J., 1965. MR 31 \#2504.

14. A. Friedman, Partial differential equations, Holt, Rinehart and Winston, New York, 1969.

15. F. John, Plane waves and spherical means applied to partial differential equations, Interscience, New York, 1955. MR 17, 746.

16. - Derivatives of solutions of linear elliptic partial differential equations, Contributions to the Theory of Partial Differential Equations, Ann. of Math. Studies, no. 33, Princeton Univ. Press, Princeton, N.J., 1954, pp. 53-61. MR 16, 706. 
17. T. Kato, Perturbation theory for linear operators, Die Grundlehren der math. Wissenschaften, Band 132, Springer-Verlag, New York, 1966. MR 34 \#3324.

18. J.-L. Lions, Perturbations singulières dans les problèmes aux limites et en contrôles optimal, Lecture Notes in Math., vol. 323, Springer-Verlag, Berlin and New York, 1973.

19. R. B. Davis, Asymptotic solutions of the first boundary value problem for a fourth-order elliptic partial differential equation, J. Rational Mech. Anal. 5 (1956), 605-620. MR 17, 1214.

20. C. Bardos, Prolongements maximaux positifs d'opérateurs positifs et problèmes de perturbations singulières, Séminaire Goulaouic-Schwartz, 1971-72, XXVI 1-12.

21. C. Bardos, D. Brézis and H. Brézis, Perturbations singulières et prolongements maximaux d'opérateurs positifs, Arch. Rational Mech. Anal. 53 (1973-74), 69-100.

22. Z. Schuss, Singular perturbations and the transition from thin plate to membrane (to appear).

23. P. Fife, Non-linear deflection of thin elastic plates under tension, Comm. Pure Appl. Math. 14 (1961), 81-112. MR 23 \#B1735.

24. F. John, The transition from thin plate to membrane in the case of a plate under uniform tension, Continuum Mechanics and Related Problems of Analysis, Moscow, 1972, pp. 193-201.

25. - The transition from thin plate to membrane in the equations of v.Karman-Föppl, Rend. Mat. (to appear).

26. S. Agmon, A. Douglis and L. Nirenberg, Estimates near the boundary for solutions of elliptic partial differential equations satisfying general boundary conditions. I, II, Comm. Pure Appl. Math. 12 (1959), 623-727; ibid. 17 (1964), 35-92. MR 23 \#A2610; 28 \#5252.

27. I. S. Sokolnikoff, Mathematical theory of elasticity, 2nd ed., McGraw-Hill, New York, 1956. MR 17, 800.

28. R. A. Toupin, Saint-Venant's principle, Arch. Rational Mech. Anal. 18 (1965), 83-96. MR $30 \# 2725$.

29. J. K. Knowles, On Saint-Venant's principle in the two-dimensional linear theory of elasticity, Arch. Rational Mech. Anal. 21 (1965), 1-22. MR 32 \#4930.

30. J. J. Roseman, The principle of Saint-Venant in linear and non-linear plane elasticity, Arch. Rational Mech. Anal. 26 (1967), 142-162. MR 35 \#7630.

31. F. John, Estimates for the derivatives of the stresses in a thin shell and interior shell equations, Comm. Pure Appl. Math. 18 (1965), 235-267. MR 30 \#5569.

32. A. Friedman, Generalized functions and partial differential equations, Prentice-Hall, Englewood Cliffs, N.J., 1963. MR 29 \#2672.

33. Neil Berger, Estimates for the derivatives of the velocity and pressure in shallow water flow and approximate shallow water equations, SIAM J. Appl. Math. 27 (1974), 256-280.

34. I. N. Sneddon and D. S. Berry, The classical theory of elasticity, Handbuch der Physik, vol. VI, Springer-Verlag, Berlin.

35. C. A. T. Truesdell and W. Noll, The non-linear field theories of mechanics, Handbuch der Physik, Band III/3, Springer-Verlag, Berlin, 1965, pp. 1-602. MR 33 \#2030.

36. F. John, Rotation and strain, Comm. Pure Appl. Math. 14 (1961), 391-413. MR 25 \#1672.

37. —_ Quasi-isometric mappings, Seminari 1962/63 Anal. Alg. Geom. e Topol., vol. 2, Ist. Naz. Alta Mat., Edizione Cremonese, Rome, 1965, pp. 462-473. MR 32 \#8315.

38. - Quasi-isometric mappings. I, Comm. Pure Appl. Math. 21 (1968), 77-110. MR 36 \#5716.

39 F. John and L. Nirenberg, On functions of bounded mean oscillation, Comm. Pure Appl. Math. 14 (1961), 415-426. MR 24 \#A1348.

40. J. Moser, On Harnack's theorem of elliptic differential equations, Comm. Pure Appl. Math. 14 (1961), 577-591. MR 28 \#2356.

41. N. G. Meyers, Mean oscillation over cubes and Hölder continuity, Proc. Amer. Math. Soc. 15 (1964), 717-721. MR 29 \#5969.

42. S. Spanne, Some function spaces defined using the mean oscillation over cubes, Ann. Scuola Norm. Sup. Pisa (3) 19 (1965), 593-608. MR 32 \#8140.

43. N. S. Trudinger, On imbeddings into Orlicz spaces and some applications, J. Math. Mech. 17 (1967), 473-483. MR 35 \#7121. 
44. E. M. Stein, Singular integrals and differentiability properties of functions, Princeton Math. Ser., no. 30, Princeton Univ. Press, Princeton, N.J., 1970. MR 44 \#7280.

45. C. Fefferman and E. M. Stein, $H^{p}$ spaces of several variables, Acta. Math. 129 (1972), 137-193.

46. F. W. Gehring, The $L^{p}$-integrability of the partial derivatives of a quasiconformal mapping, Acta Math. 130 (1973), 265-277.

47. H. M. Reimann, Functions of bounded mean oscillation and quasiconformal mappings, Comment. Math. Helv. 49 (1974), 260-276.

48. F. John, Continuous dependence on data for solutions of partial differential equations with a prescribed bound, Comm. Pure Appl. Math. 13 (1960), 551-585. MR 24 \#A317.

Department of Mathematics, Courant Institute of Mathematical Sciences; New YoRK UNIVERSITY, NEW YORK, NEW YORK 10012 